\title{
Functional Dyspepsia and Chronic Gastritis Associated with Enteroviruses
}

\author{
John K. Chia, Andrew Y. Chia, David Wang, Rabiha El-Habbal \\ EV Med Research LLC, Lomita, USA \\ Email: evmed@sbcglobal.net
}

Received 26 February 2015; accepted 3 April 2015; published 9 April 2015

Copyright (C) 2015 by authors and Scientific Research Publishing Inc.

This work is licensed under the Creative Commons Attribution International License (CC BY). http://creativecommons.org/licenses/by/4.0/

(c) (i) Open Access

\begin{abstract}
After decades of research, functional dyspepsia (FD) remains one of the most elusive gastrointestinal disorders. Endoscopic appearance of mild inflammation of the gastric mucosa without ulceration and microscopic evidence of mild chronic inflammation are often considered as normal findings since no etiology could be found other than $\mathrm{H}$. pylori. Enteroviruses infect the gastrointestinal tract and have been shown to persist in the stomach of symptomatic patients with myalgic encephalomyelitis/chronic fatigue syndrome (ME/CFS). In this study, we evaluated FD patients with and without the diagnosis of ME/CFS, and were able to support the viral protein staining with finding of double-stranded RNA in $63 \%$ of the same stomach biopsies by immunoperoxidase staining. Furthermore, we clarified the possible cross-reaction with creatine kinase brain subtype $\left(\mathrm{CK}_{\mathrm{B}}\right)$, present in parietal cells, using antibody competition experiments and western blot analysis of stomach proteins. Viral protein+ and dsRNA+ biopsies were infectious in SCID mice. More research is needed to elucidate the mechanism of enterovirus infection of the stomach associated with FD and chronic gastritis.
\end{abstract}

\section{Keywords}

Functional Dyspepsia, Chronic Gastritis, Enterovirus, Double Stranded RNA

\section{Introduction}

Functional dyspepsia (FD) is one of the most prevalent and elusive disorders of the gastrointestinal (GI) tract. It is estimated that $20 \%-40 \%$ of general population would have some symptoms of dyspepsia and most patients are believed to have FD [1] [2]. When severe, the patients present with epigastric pain, debilitating post-prandial nausea and bloating, vomiting, early satiety requiring frequent visits to physician's offices, emergency rooms and hospitalizations. 
The etiology for FD remains unknown despite decades of research [2]. The symptoms are believed to be due to abnormal function of the muscles and/or nerves supplying the gastrointestinal tract. The function of local nerve network is further modulated by the central nervous system via the vagus nerve and sympathetic trunk. Abnormality or injury in the local nerves plexus, vagus nerve, spinal cord and brain can result in significant dysfunction of the GI tract. Either abnormal sensory signals coming from GI organs or excessive motor nerve output from the brain could result in the symptoms of FD and IBS. Therefore, a disease such as gastritis or colitis may cause changes in the sensitivity of the nerves or processing centers of the GI tract.

The term gastritis was first coined in 1728 by Dr. Georg Stahl describing the inflamed inner lining of the stomach [3]. Since the discovery of $H$. pylori in 1982 [4], identification, description and classification of a multitude of different gastritides were established [3]. Chemical gastritis can be caused by bile, NSAID, alcohol, aspirin and chemotherapeutic agents, and has minimal or no inflammation in the biopsies. Autoimmune and allergic mechanisms have also been proposed for the inflammatory response in some cases [5]-[8].

The most studied pathogen of the stomach is $H$. pylori. However, acute and persistent chronic $H$. pylori infections usually do not cause symptoms despite clinical and pathologic evidence of active inflammation of the stomach. Controlled treatment studies demonstrated that cases of FD associated with mild gastritis and $H$. pylori infections had shown variable response to anti-H. pylori treatment and eradication [2] [9]. Empiric treatment for undocumented $H$. pylori in FD patients in area of low prevalence $(<5 \%)$ is not recommended due to the low likelihood of achieving symptomatic improvement and development of antibiotic resistance [2].

Although $H$. pylori is the "most common" known cause of chronic gastritis, it is only found in less than $5 \%$ of the stomach biopsies taken from our patients with FD (see below). Often, the patients were informed they do not have a cause of the chronic gastritis seen endoscopically or by microscopic examination, and therefore the complaints would fall into the category of functional disorders.

Although persistent symptoms can follow acute viral gastroenteritis, chronic enteroviral infection of the GI tract has not been adequately studied in patients with FD. In the course of studying enterovirus infection of GI tracts of patients with myalgic encephalomyelitis/chronic fatigue syndrome (ME/CFS), our laboratory documented chronic persistent infection of stomach, small bowel and colon in these patients [10] [11]. We identified enterovirus infection in the stomach of patients presenting with acute onset of severe epigastric pain, vomiting and found to have mild antral gastritis by endoscopic and microscopic examinations; many patients developed chronic symptoms of FD [12]. Similarly, we found enterovirus infection of the stomach in patients with functional dyspepsia without CFS [13], and further supported our preliminary finding by demonstrating double stranded RNA in the stomach biopsies of these patients.

\section{Materials and Methods}

\subsection{Patient Selection}

Between 2006 and 2012, 416 patients (85\% female, 15\% male; 75\% Caucasian, 13\% Hispanic, 10\% Asian; age $44 \pm 16)$ fulfilling the necessary criteria for ME/CFS as established by the international working group on ME/CFS [14] had EGD and biopsies of stomach, as described [10]. Patients with diagnosis of functional dyspepsia presenting with symptoms of epigastric pain, burning, nausea and post-prandial bloating for more than 6 months, without a diagnosis of ME/CFS, underwent the same procedures ( $\mathrm{n}=42,74 \%$ female, $26 \%$ male, age $38 \pm 10$ ). The control subjects included asymptomatic volunteers ( $\mathrm{n}=12$; 9 female, 3 males; age $35 \pm 8$ ), patient with unexplained anemia $(n=7)$, NSAID-induced gastritis $(n=3)$. Coded unstained slides for FD patients $(n=$ 24) and normal volunteer ( $n=25,60 \%$ female, $40 \%$ male) were obtained from Dr. El Serag, Baylor University Medical Center, Houston, Texas [10] [12] [13]. The experimental protocol was approved by the Institution Review Board of Providence Little Company of Mary Hospital, Torrance, California.

\subsection{Immunoperoxidase Staining (IPOX)}

IPOX of the paraffin-embedded tissues was performed using enterovirus-specific murine monoclonal antibody (25 - $35 \mu \mathrm{L}$ of 5D8/1 at 1:1000 dilution, Dako, Carpenteria, CA, USA) directed against a highly conserved, nonconformational epitope of viral capsid protein 1 (VP1), or a mAb specific for dsRNA (25 - 35 $\mu \mathrm{L}$ of J2 at 1:500 dilution, English \& Scientific consulting, Hungry, ref. [11] [12]. Briefly, the deparaffinized, hydrated biopsy specimens were treated with $3 \% \mathrm{H}_{2} \mathrm{O}_{2}$ to remove endogenous peroxidase activity, undergo heat-induced epitope 
retrieval, blocked with goat serum, treated with biotin-avidin to remove endogenous biotin in gastric tissue, then incubate sequentially with murine mAb, biotinylated goat anti-mouse antibody (Vectastain ABC reagent, Vector Laboratory), before developing with DAB chromogen. Hematoxylin is used as counter stain. The concentration of the mAB was optimized by titration, and all the tests were performed by hands since automated stainer tend to over-stain the slides even with more dilution of the primary antibody.

Since our publication on viral protein staining of stomach biopsies [10], another published study questioned the validity of enteroviral VP1 staining of pancreatic islet cells using 5D8/1, and demonstrated that antibody directed against Creatine kinase brain subtype $\left(\mathrm{CK}_{\mathrm{B}}\right)$ and ATPse could cross-react with the same epitope as 5D8/1 [15]. Rabbit polyclonal antibody raised against the N-terminus 1-100 amino acid of $\mathrm{CK}_{B}$ (R anti-CK $\mathrm{C}_{\mathrm{B}} \mathrm{N}$, ab38212), rabbit monoclonal IgG against the $\mathrm{C}$-terminus of $\mathrm{CK}_{\mathrm{B}}\left(\mathrm{R}\right.$ anti-CK $\mathrm{C}_{\mathrm{B}}-\mathrm{C}$, ab92452) were purchased from Abcam, Cambridge, MA, and rabbit polyclonal antibody directed against ATPse (R anti-ATPse, HPA001520) was obtained from Sigma. St Luis MO.

32 stomach biopsy specimens were treated with and without prior incubation with 1:100 dilution of the rabbit anti-CK $\mathrm{C}_{\mathrm{B}} \mathrm{N}$, and anti-ATPase for 30 minutes before adding 5D8/1 for EV VP1 stain. 15 of the 32 specimens were also blocked with 1:500 dilution of anti-CK $\mathrm{CK}_{\mathrm{B}}-\mathrm{C}$ for 30 minutes before adding 5D8/1.

In order to demonstrate the specificity of the dsRNA staining procedure, we pre-incubated selected dsRNA+ stomach samples with and without E. coli RNAse III (life technology, catalog \# AM2290) in reaction buffer at $37^{\circ} \mathrm{C}$ for one hour before performing dsRNA staining.

\subsection{Polyacrylamide Gel Electrophoresis and Western Blot Analysis}

(PAGE/WB) was done to elucidate the proteins involved in antibody binding. Lysates of Coxsackievirus $\mathrm{B}_{3}$ $\left(\mathrm{CVB}_{3}\right)$ infected tissue culture (lane 1), uninfected Hela cells (lane 2), uninfected Hep 2 cell (lane 3), uninfected human $\mathrm{CaCO} 2$ cells (lane 4), human brain tissues from occipital lobe (lane 5) and cerebellum (lane 6) and 3 VP1+ and dsRNA+ stomach biopsies (lanes 7, 8, 9) were separated with Novex Bolt 4\% - 12\% Bis-Tris plus mini-gel and dry blotted unto nitrocellulose membrane using Transblot Turbo (Bio-Rad), then the nitrocellulose membrane was sequentially incubated with 5D8/1 antibody at 1:1500 dilution, 1:3000 dilution of Goat antimouse IgG (highly absorbed) in Ibind (Novex, life technologies) and developed with DAB chromogen, according to manufacturer's instruction.

\section{Results}

At the time of EGD, careful inspection of the stomach of FD patients with or without ME/CFS, demonstrated focal or rarely diffuse, erythematous areas in the antrum in approximately $80 \%$ of patients, and less often in the body/fundus of the stomach. Microscopic examinations demonstrated minimal or mild chronic inflammation in more than $95 \%$ of biopsy samples but $H$. pylori was identified in less than $5 \%$ of samples by immunochemical staining.

Immunoperoxidase staining demonstrated enterovirus VP1 in 343/416 (82\%) and 53/66 (83\%) of the stomach biopsies from FD patients with and without ME/CFS, respectively, and dsRNA in 268/ 416 (64\%) and 41/65 (63\%) in the two patient cohorts, respectively (Figure 1). 9/47 (19\%) and 5/46 (11\%) of the controls stained positive for VP1 and dsRNA, respectively ( $\mathrm{p}<0.01, \chi^{2}$ test with Yates correction).

VP1 was detected more often than dsRNA in stomach biopsy specimens from ME/CFS and FD patients. 43\%, 28\%, 29\% of dsRNA-negative specimens showed VP1-negative (double-negative), 1+ VP1 and 2+ VP1 staining, respectively. Rarely, VP1-negative specimens stained strongly positive for double-stranded RNA. 5D8/1 could only detect VP1 of $80 \%$ of the enteroviruses [17], and few VP1-negative specimens could be detected by dsRNA staining.

The dsRNA-specific antibody usually identified the viral RNA in cells located in the more basal part of the biopsies as compared to the VP1 staining (Figure 1(A), Figure 1(B)). Pre-treatment with RNase III of selective samples diminished or abolished the dsRNA staining (Figure 2(A), Figure 2(B)); $4 \times$ concentration of enzyme and 2× incubation period were required for specimens from sicker patients (Figure 2(C), Figure 2(D)).

Comparison of VP1staining with or without prior blocking with anti-CK $\mathrm{C}_{\mathrm{B}} \mathrm{N}$ and anti-ATPse antibody showed positive results in 31/32 and 32/32 biopsy specimens, respectively, with a concordance rate of 97\%. 10/32 specimens had lighter staining with 5D8/1 when blocked with the two competing antibodies (Figure 3), and only one specimen was completely blocked by the two antibodies. Both rabbit polyclonal anti- $\mathrm{CK}_{\mathrm{B}}-\mathrm{N}$ and monoclonal 

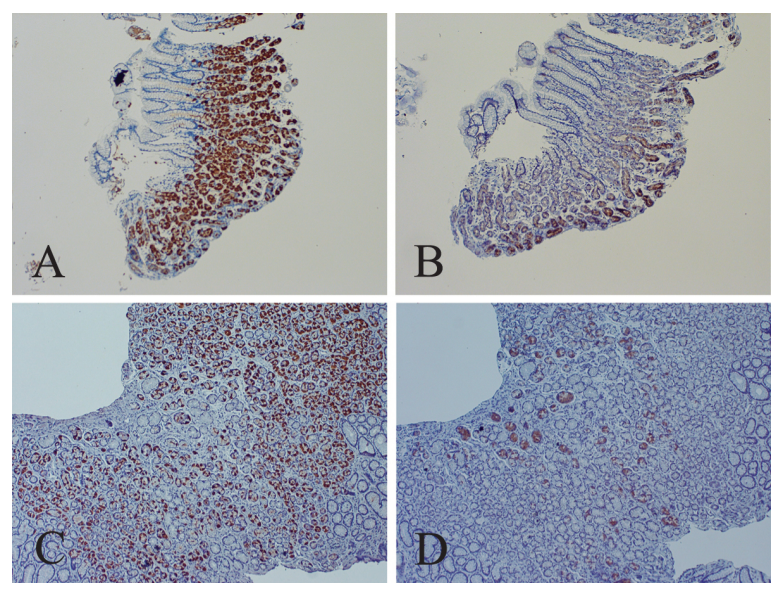

Figure 1. Staining for VP1 and dsRNA in stomach biopsies of two functional dyspepsia (FD) patients. Panels A and B demonstrate positive staining for VP 1 with 5D8/1 mAb and dsRNA with J2 mAb, respectively for patient 1 (brown colored cells, $100 \times$ magnification); panels $\mathrm{C}$ and $\mathrm{D}$ showed the same for patient 2.
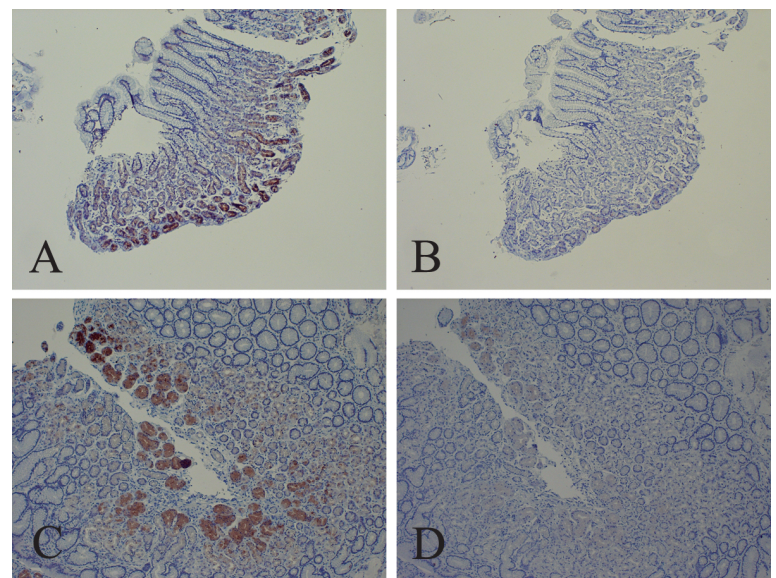

Figure 2. DsRNA staining of stomach biopsies with and without RNAse III digestion. Panels A and C depict stomach biopsies from FD patients stained for dsRNA without RNAse III digestion (100× magnification); panels B and D represent the same specimens with prior RNAse III digestion before staining with $\mathrm{J} 2 \mathrm{mAb}$.
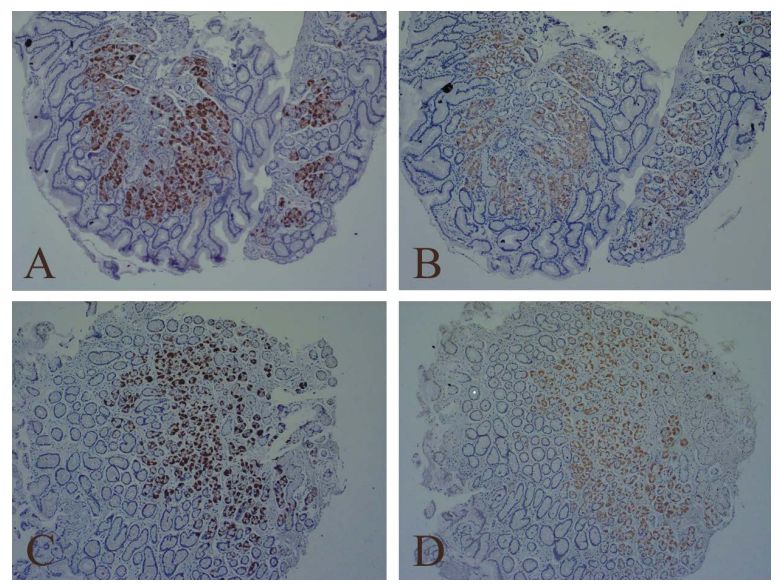

Figure 3. Competition experiments with rabbit anti-CK $\mathrm{B}_{\mathrm{B}}$ antibody N-terminus and C-terminus. A and C panels represent stomach biopsies of two patients pre-incubated with $\mathrm{R}$ anti-CK $\mathrm{B}-\mathrm{C}$; and $\mathrm{B}$ and $\mathrm{D}$ are the same slides pre-incubated with $\mathrm{R}$ anti-CK $\mathrm{C}_{\mathrm{B}} \mathrm{N}$ before IPOX with 5D8/1 (100× magnification). The slides stained with or without pre-incubation with $\mathrm{R}$ anti-CK $-C$ showed the same staining and not repeated in the figure. 
anti-CK $\mathrm{B}_{\mathrm{B}} \mathrm{C}$ stained the parietal cells, similar to 5D8/1, when developed with HRP-conjugated goat anti-rabbit antibody but not with HRP-conjugated goat anti-mouse antibody. Anti-ATPase antibodies stained the gastric tissues more diffusely (Picture not shown). When the slides were first incubated with anti-CK $\mathrm{B}_{\mathrm{B}} \mathrm{C}$ before 5D8/1, no inhibition of staining was seen.

PAGE/WB of Hela cells and brain tissue demonstrated positive band at $46 \mathrm{kDa}$, which was also seen when the blots were separately developed with anti-CK $-\mathrm{N}$ or $-\mathrm{C}$, consistent with $\mathrm{CK}_{\mathrm{B}}$. The lower molecular weight bands seen in lane 7 - 9 (40 - $42 \mathrm{kDa})$ of $3 \mathrm{VP1}+$ and dsRNA+ stomach biopsy lysate were clearly different from the $\mathrm{CK}_{\mathrm{B}}$ band, but appeared to be a larger protein than VP1 isolated from Coxsackievirus $\mathrm{B}_{3}$ culture (35 kDa).

21/23 (91\%) of stomach biopsies previously tested positive for EV RNA by RT-PCR or had grown non-cytopathic virus in BGMK-DAF cells were positive for dsRNA by IPOX. To date, 30/33 (91\%) of the RNA+ biopsy samples were positive for dsRNA by IPOX.

\section{Discussion}

The results of enterovirus protein and dsRNA staining were identical for patients with functional dyspepsia/ chronic gastritis with or without ME/CFS; and significantly more positive compared to the control subjects.

Previous paper described co-localization and functional coupling of creatine kinase B and gastric $\mathrm{H}+/ \mathrm{K}+-$ ATPse on the apical membrane and the tubulovesicular system of parietal cells [18]. $\mathrm{CK}_{\mathrm{B}}$ is expressed at high levels in gastric parietal cells of human, mice and rabbits. Hansson's study demonstrated cross-reactivity of 5D8/1 with comparable epitope in the N-terminus of CKb and ATPase [15]. We perform competition experiments with antibody directed against the similar epitope on the N-terminus and C-terminus of $\mathrm{CK}_{\mathrm{B}}$ and also ATPse. Although blocking experiments with anti-CK $\mathrm{B}_{\mathrm{B}}-\mathrm{C}$ did not significantly inhibit the binding of 5D8/1 to parietal cells, anti-CK $\mathrm{B}_{\mathrm{B}} \mathrm{N}$ and anti-ATPse antibodies did partially inhibit the binding in 10/32 samples. AntiATPse alone did not block 5D8/1 binding, similar to what was found in other study [16]. The cross-reactive epitope of $\mathrm{CK}_{\mathrm{B}}$ with antibody 5D8/1 is located in the first 100 amino acids of the N-terminus [15], and therefore, it is not surprising that low dilution of polyclonal anti-CK $\mathrm{B}_{\mathrm{B}} \mathrm{N}$-terminus antibody could partially block the binding of 5D8/1. However, the monoclonal antibody raised against the C-terminus of the same molecule did not block the binding of 5D/81, and in some cases, actually enhanced the staining by 5D8/1. If 5D8/1 is binding to $\mathrm{CK}_{\mathrm{B}}$ in the parietal cells, then the antibody directed against the C-terminus of a relatively small molecule should be able to block the binding of 5D8/1 as well.

In order to clarify this issue further, we performed PAGE/WB analysis of 3 stomach biopsies markedly positive for VP1 protein and dsRNA and used Hela cells and brain tissues as positive controls for $\mathrm{CK}_{\mathrm{B}}$ (Figure 4). The positive bands around $46 \mathrm{kDa}$ was recognized by $5 \mathrm{D} 8 / 1$, rabbit anti-CK $\mathrm{B}-\mathrm{N}$ and $-\mathrm{C}$, but the lower band around 40 - $42 \mathrm{kDa}$ of the stomach biopsies was recognized by 5D8/1and not blocked by pre-incubation with rabbit anti-CK $\mathrm{B}_{\mathrm{B}} \mathrm{N}$-terminus or C-terminus (data not shown). The size of the viral protein from the stomach biopsies was larger than VP1 from a pure $\mathrm{CVB}_{3}$ culture. The significance of the finding is unknown and will required additional investigations.

Finding dsRNA in the stomach biopsies not only supported the VP1 staining data but also offered a possible mechanism of viral persistence. Viral RNA is required for protein translation, and finding both RNA and protein suggests there is active viral replication and protein synthesis.

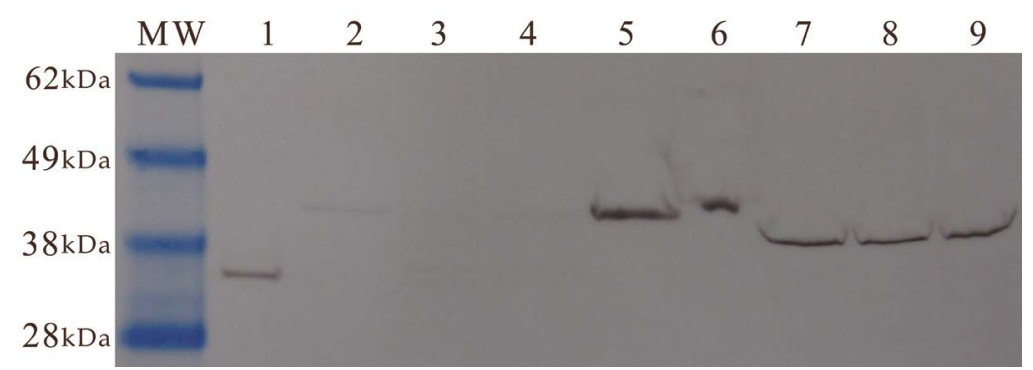

Figure 4. PAGE/WB analysis of $\mathrm{CK}_{\mathrm{B}}$ and viral proteins from Coxsackievirus $\mathrm{B}_{3}$-infected cells (lane 1), uninfected Hela cells, Hep 2 cells, CaCo2 colon carcinoma cells (lanes 2, 3, 4), human brain specimens (lanes 5, 6) and VP1+ and dsRNA+ stomach biopsy specimens (lane 7, 8, 9), incubated with 5D8/1. $\mathrm{CK}_{\mathrm{b}}$ band was $46 \mathrm{kDa}$ and viral protein from stomach biopsy at $40-42 \mathrm{kDa}$. 
Pre-treatment of dsRNA+ samples with RNAse III, specific for degrading dsRNA, decreased or abolished the binding of dsRNA-specific antibody confirming specificity of the staining test. Severely ill ME/CFS patients had more dsRNA in the stomach biopsies since higher concentration of RNAse III and longer incubation periods were required to decrease immuno-reactivity of the biopsy specimens.

91\% of stomach biopsy specimens previously tested positive for EV RNA by RT-PCR, and had grown "noncytopathic virus" were dsRNA+ by staining, confirming the immunoreactivity is most likely from enterovirus RNA in the cells. The staining procedure is more sensitive and easier to perform as compared to the tedious RNA extraction/RT-PCR and culture technique [10] [11].

The amount of inflammatory cells around the virus-infected stomach biopsies was minimal in most cases. Our preliminary, unpublished immunochemical studies showed more CD8+ T lymphocytes around the virus-infected parietal cells in patient with the most FD symptoms, as compared to patient with minimal symptoms. The exact mechanism for the stomach complaints and endoscopic evidence of gastritis in these patients remains unknown and will require further investigations.

When injected into SCID mice, the homogenates of VP1+, dsRNA+ specimens resulted in infection of the spleens of majority of mice, and 2 mice died in 2 weeks, but boiled stomach homogenate failed to infect the mice. IPOX demonstrated positive VP1 staining in the cytoplasm of splenic cells (manuscript in preparation).

Taken together, these findings suggest that persistence of viral genome in the stomach tissue is in the form of dsRNA, and that expression of enteroviral protein by the infected cells is consistent with persistent infection without lysis of cells. One obvious question emerges with these observations is why the infected cells failed to undergo apoptosis in the presence of intracellular viral dsRNA [19]. Future experiments will need to explore the mechanism of viral persistence, which has been demonstrated in muscle, heart and brain of animal and in human [20]. Although the 5' highly-conserved nucleotide sequences of enteroviruses were found and published [10], successful sequencing of complete viral RNA genome found in the stomach biopsies will provide even stronger evidence for persistent enterovirus infection and further elucidate the mechanism of viral persistence without apoptosis.

\section{Conclusion}

In summary, our studies provided evidence for the pathogenic role of enteroviral dsRNA in the stomach biopsies of patients who had FD/chronic gastritis with and without ME/CFS. Much more research will be needed to define the mechanism of virus-mediated tissue injury and host responses. Development of antiviral therapy against enteroviruses and/or dsRNA cannot be overemphasized, and the importance of enteroviruses in FD/chronic gastritis can only be realized with a randomized, placebo-controlled antiviral drug trial.

\section{Acknowledgements}

We are indebted to the many gastroenterologists in our local areas who performed EGD and biopsies on the study patients.

\section{References}

[1] Camilleri, M., Dubois, D., Coulie, B., et al. (2005) Prevalence and Socioeconomic Impact of Upper Gastrointestinal Disorders in the United States: Results of the US Upper Gastrointestinal Study. Clinical Gastroenterology and Hepatology, 3, 543-552. http://dx.doi.org/10.1016/S1542-3565(05)00153-9

[2] Lacy, B.E., Talley, N.J., Locke 3rd, G.R., et al. (2012) Review Article: Current Treatment Options and Management of Functional Dyspepsia. Alimentary Pharmacology \& Therapeutics, 36, 3-15. http://dx.doi.org/10.1111/j.1365-2036.2012.05128.x

[3] Dixon, M.F., Genta, R.M., Yardley, J.H. and Correa, P. (1996) Classification and Grading of Gastritis. The Updated Sydney System. International Workshop on the Histopathology of Gastritis, Houston 1994. The American Journal of Surgical Pathology, 20, 1161-1181. http://dx.doi.org/10.1097/00000478-199610000-00001

[4] Warren, J.R. and Marshall, B. (1983) Unidentified Curved Bacilli on Gastric Epithelium in Active Chronic Gastritis. Lancet, 1, 1273-1275.

[5] Gao, L., Weck, M.N., Stegmaier, C., Rothenbacher, D. and Brenner, H. (2009) Alcohol Consumption and Chronic Atrophic Gastritis: Population-Based Study among 9,444 Older Adults from Germany. International Journal of Cancer, 125, 2918-2922. http://dx.doi.org/10.1002/ijc.24618 
[6] Sepulveda, A.R. and Patil, M. (2008) Practical Approach to the Pathologic Diagnosis of Gastritis. Archives of Pathology \& Laboratory Medicine, 132, 1586-1593.

[7] Neumann, W.L., Coss, E., Rugge, M. and Genta, R.M. (2013) Autoimmune Atrophic Gastritis-Pathogenesis, Pathology and Management. Nature Reviews Gastroenterology \& Hepatology, S10, 529-541. http://dx.doi.org/10.1038/nrgastro.2013.101

[8] Wu, T.T. and Hamilton, S.R. (1999) Lymphocytic Gastritis: Association with Etiology and Topology. The American Journal of Surgical Pathology, 23, 153-158. http://dx.doi.org/10.1097/00000478-199902000-00003

[9] Zhao, B., Zhao, J., Cheng, W.F., et al. (2014) Efficacy of Helicobacter pylori Eradication Therapy on Functional Dyspepsia: A Meta-Analysis of Randomized Controlled Studies with 12-Month Follow-Up. Journal of Clinical Gastroenterology, 48, 241-247. http://dx.doi.org/10.1097/MCG.0b013e31829f2e25

[10] Chia, J. and Chia, A. (2008) Chronic Fatigue Syndrome Is Associated with Chronic Enterovirus Infection of the Stomach. Journal of Clinical Pathology, 61, 43-48. http://dx.doi.org/10.1136/jcp.2007.050054

[11] Chia, J., Chia, A., Voeller, M., Lee, T. and Chang, R. (2010) Acute Enterovirus Infection Followed by Myalgic Encephalomyelitis/Chronic Fatigue Syndrome (ME/CFS) and Viral Persistence. Journal of Clinical Pathology, 63, 165-168. http://dx.doi.org/10.1136/jcp.2009.070466

[12] Chia, J. and Chia, A. (2010) Acute Gastritis Associated with Enterovirus Infection. Archives of Pathology and Laboratory Medicine, 134, 16-17.

[13] El-Serag, H., Chia, J. and Chia, A. (2007) Enterovirus Infection of the Stomach Is Associated with Functional Dyspepsia. Abstract \#448, Digestive Disease Week, Washington DC, 21 May 2007.

[14] Reeves, W.C., Lloyd, A., Vernon, S.D., Klimas, N., Jason, L.A., Bleijenberg, G., et al. (2003) Identification of Ambiguities in the 1994 Chronic Fatigue Syndrome Research Case Definition and Recommendations for Resolution. BMC Health Services Research, 3, 25-29. http://dx.doi.org/10.1186/1472-6963-3-25

[15] Hansson, S.F., Korsgren, S., Pontén, F. and Korsgren, O. (2013) Enteroviruses and the Pathogenesis of Type 1 Diabetes Revisited: Cross-Reactivity of Enterovirus Capsid Protein (VP1) Antibodies with Human Mitochondrial Proteins. Journal of Pathology, 229, 719-728. http://dx.doi.org/10.1002/path.4166

[16] Richardson, S.J., Leete, P., Dhayal, S., Russell, M.A., Oikarinen, M., Laiho, J.E., et al. (2014) Evaluation of the Fidelity of Immunolabelling Obtained with Clone 5D8/1, a Monoclonal Antibody Directed against the Enteroviral Capsid Protein, VP1, in Human Pancreas. Diabetologia, 57, 392-401. http://dx.doi.org/10.1007/s00125-013-3094-7

[17] Yousef, G.E., Brown, I.N. and Mowbray, J.F. (1987) Derivation and Biochemical Characterization of an Enterovirus Group-Specific Monoclonal Antibody. Intervirology, 28, 163-170.

[18] Sistermans, E.A., Klaassen, C.H., Peters, W., Swarts, H.G.P., Jap, P.H.K., et al. (1995) Co-Localization and Functional Coupling of Creatine Kinase B and Gastric $\mathrm{H}^{+} / \mathrm{K}^{+}$-ATPase on the Apical Membrane and the Tubulovesicular System of Parietal Cells. Biochemical Journal, 311, 445-451.

[19] Colli, M.L., Nogueira, T.C., Allagnat, F., Cunha, D.A., Gurzov, E.N., Cardozo, A.K., et al. (2011) Exposure to the Viral By-Product dsRNA or Coxsackievirus B5 Triggers Pancreatic Beta Cell Apoptosis via a Bim/McL-1 Imbalance. PLoS Pathogens, 7, e1002267. http://dx.doi.org/10.1371/journal.ppat.1002267

[20] Chia, J. (2005) The Role of Enterovirus in the Chronic Fatigue Syndrome. Journal of Clinical Pathology, 58, 11261132. http://dx.doi.org/10.1136/jcp.2004.020255 